\title{
Heat Diffusion - Searching for the Accurate Modeling
}

\author{
Malgorzata Langer ${ }^{1}$, Janusz Wozny ${ }^{1}$, Malgorzata Jakubowska ${ }^{2}$, and Zbigniew Lisik ${ }^{1}$ \\ ${ }^{1}$ Institute of Electronics, Technical University of Lodz, \\ 90-924 Lodz, ul. Wolczanska 211/215, Poland \\ \{malgorzata.langer, jwozny, zbigniew.lisik\}@p.lodz.pl \\ ${ }^{2}$ Institute of Electronic Materials Technology, Wolczynska Str. 133 \\ 01-919 Warsaw, Poland \\ malgorzata.jakubowska@itme.edu.pl
}

\begin{abstract}
The authors study three approaches which allow to model the steady state heat conduction in a $2 \mathrm{D}$ multiphase composite. The subject under investigating is the thermal conductivity of a c-BN composite thick film with possible inclusions of air bubbles. To define the thermal conductivity we have utilized (i) a commercial program ANSYS, for which a random structure has been externally generated, (ii) a cellular automata (CA) based model and (iii) a modified cellular automata based model where we have taken into account a thermal contact resistance between adjacent grains of c-BN.
\end{abstract}

\section{Introduction}

Up-to-now the whole electronics, that is based on silicon can work in the low temperature regime (max. $473 \mathrm{~K}$ ), and yet one should be aware that it will be soon when the device should meet the demand to take as huge power density, as 400 $\mathrm{W} / \mathrm{cm}^{2}$ [1]. That is why there are intensive works kept on designing new and improving known materials with extremely high heat conductivity to be capable taking such values over. The good and verified thermal model for mixtures, conglomerates and other composites becomes an issue. Such materials as cubic boron nitride (c-BN), gallium nitride $(\mathrm{GaN})$, silicon carbide ( $\mathrm{SiC})$, and some others are to widen the temperature range for electronic devices. One hopes that the mentioned materials may allow increasing the temperature range even up to about $900 \mathrm{~K}$. The Table 1 introduces cubic boron nitride features among which the good heat conductivity, electrical insulation capabilities, the resistance against high temperature and chemical agents' impact make one being interested in this material. As with CVD (Chemical Vapor Deposition) or PVD (Physical Vapor Deposition) it is impossible obtaining layers thicker than $3000 \AA$ (they delaminate [2]) and yet, it is difficult to obtain crystalline structure that is wanted in electronics, the conception of thick film technology - screen printing was realized and the c-BN films, $20-150 \mu \mathrm{m}$ thick, with a high adhesion to the substrate were deposited.

The tested films were deposited on alumina $\left(\mathrm{Al}_{2} \mathrm{O}_{3} 96 \%\right)$, with a screen printing technique through 325 mesh screen, then fired in a thick film belt furnace in $850^{\circ} \mathrm{C}$, 10 minutes. The printed thick film composition ('electronic paste'), based on c-BN is a dispersive mixture of inorganic powders in a solution of organic resins. It consists of three basic components: 
- the active phase that determines the electrical and thermal properties of the layer, i.e. the grains of cubic boron nitride, $2-5 \mu \mathrm{m}$ big, obtained with method shown in [3];

- the auxiliary phase (bonding) - bismuth-boron-silicate glass;

- the organic vehicle

The obtained film consists of c-BN grains surrounded by glass, with closed and open pores filled with air (Fig. 1)

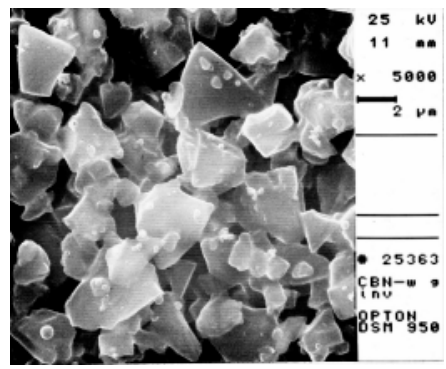

Fig. 1. SEM image with inverted contrast; a sample of the c-BN composite (magnified 5000x)

Table 1. Components and material properties of a c-BN composite sample

\begin{tabular}{|c|c|c|c|}
\hline Material & $\begin{array}{c}\text { thermal conductivity } \\
{\left[\mathbf{W} / \mathbf{m}^{\mathbf{2}} \mathbf{K}\right]}\end{array}$ & $\begin{array}{c}\text { density } \\
{\left[\mathbf{g} / \mathbf{c m}^{\mathbf{3}}\right]}\end{array}$ & $\begin{array}{c}\text { volume fraction } \\
{[\%]}\end{array}$ \\
\hline c-BN & 1300 & 3.48 & 85.67 \\
\hline glass & 12 & 6.64 & 4.32 \\
\hline air & 0.00271 & 0.0013 & 10 \\
\hline
\end{tabular}

It is obvious the mutual ratio of these phases influences the thermal conductivity of the film. The measurements for the film $100 \mu \mathrm{m}$ thick (made with the method described in [4]) result with values in the range 100-200 W/mK, depending on the c-BN grain fraction, the purity, and the volume of pores. Comparing this number to the c-BN thermal conductivity, one can see that although the thermal conductivity of c-BN is very high, the effective thermal conductivity of a composite film is much lower due to the presence of the glass, air inclusions and the fact that there are grains of c-BN only and not a continuous layer of a monocrystal. Since the gas pores are very small, one can neglect the convection and treat them as a material of low thermal conductivity. So the conductivity of the composite layer is affected by the size and the shape of grains, their orientation and the volume of c-BN phase. Since the thickness of a layer is a dozen of grains one should consider the shape, the size and the position of every grain.

There is a lack of references, where thermal modeling of composites deals with different sizes and irregular shapes of grains. For instance in [5] every particle with the high thermal conductivity is considered as a circle or sphere of randomly varying radius and position. In [6] and [7] every node represents an element with the high or 
low conductivity. No thermal resistance of contacts was considered between neighboring elements. The effective thermal conductivity of the layer was governed by the existence of percolation path, mainly. A (random) resistors network has been generated first [5], [6], [7] and then the solution was obtained with the use of various approaches.

We have gone one step lower where the heat conduction inside grains and between them should be properly modeled to get accurate effective conductivity of the film.

\section{The First Approach - FEM}

The first idea is always using a commercial software, and we have done it to calculate an effective thermal conductivity. The ANSYS software, based on the FEM (Finite Element Method), was used. As the number of grains may be higher than few dozens so we prepare a special file (we have done it using Scilab 3.0 [8], but it may be done in any other programming language). This is the script that covers input data for geometry: information about distribution function of the grain size, permissible angles between adjacent edges of a grain and the final size of a composite sample. Thus, the structure is completely stochastic. The basic assumption is that the grains cannot overlap one another. Thus, after generating, any grain is to be placed in the structure in such a location where it does not overlap with other grains and its position is as low as possible (Fig. 2 shows an example how the geometry is constructed).

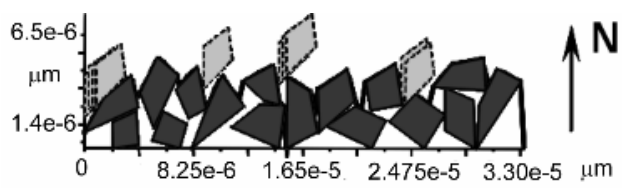

Fig. 2. A midstep of the geometry generation process. We have to place a grain in the lowest possible location.

An example of such an input file's result is presented in Fig. 3.

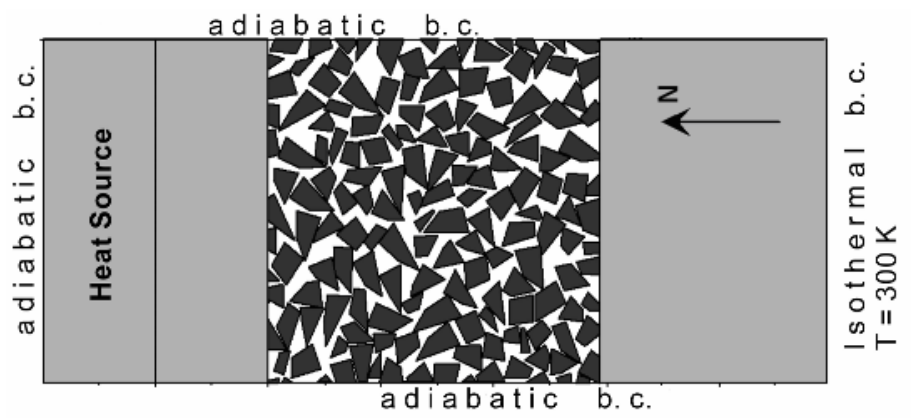

Fig. 3. The geometry used for defining the effective thermal conductivity of a composite layer 
Additional (grey) layers work as buffers between the film and the boundary conditions and guarantee a space for a heat source. Setting boundary conditions as presented in Fig. 3 assures that the entire heat flux must flow trough the composite layer. The task for ANSYS is to mesh the domain, to solve it, and to export some results Fig. 4

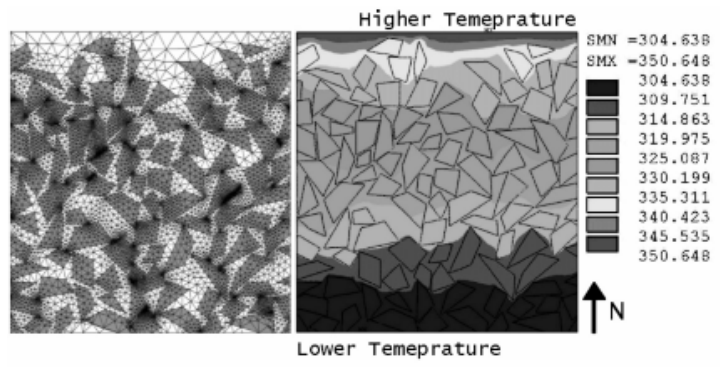

Fig. 4. The mesh and temperature distribution of the composite layer

Since one knows the dissipated power, dimensions of the film and average temperatures on both sides of it, one can calculate its effective thermal conductivity. For the film in Fig. 4 the volume fraction of c-BN was ca. $50 \%$ and no air was considered. The effective thermal conductivity amounts to ca. $64 \mathrm{Wm}^{-1} \mathrm{~K}^{-1}$.

The mesh consists of $12 \cdot 10^{3}$ nodes. It took about $1 \mathrm{~min}(2800+$ Athlon PC) to obtain these results. So one can reasonably expect that also $3 \mathrm{D}$ simulation can be performed.

Of course there are some issues that must be considered. First, the presented method of generating geometry of the structure doesn't allow getting high (ca. $>70 \%$ ) $\mathrm{c}-\mathrm{BN}$ volume ratios. Then, if we get a large number of grains comparing to the glass phase, the space between them can be very narrow which will cause problems with mesh generation. Thus a case when two grains have a common edge should be also taken into account. Summarizing, a "simple" problem of creating the composite structure evolves into modeling of composite fabrication process, not discussed here. However, the presented results show that it is possible to obtain the effective thermal conductivity with this method.

\section{Standard Cellular Automata (CA) Approach}

To solve the steady-state heat diffusion differential equation there is no need to utilize any sophisticated commercial software. One can use a cellular automata, which allows to model behaviors of physical systems [9],[10], and also to mimic a diffusion process. On a homogeneous $2 \mathrm{D}$ square lattice the temperature at a given node is obtained as a mean value of temperatures at 4 neighboring nodes (1) [11]. This is enough to mimic the diffusion. When the lattice is not uniform or the environment is not homogeneous one has to use a weighted mean (1). Such a case is presented in 
Fig. 5. The space between nodes can be chosen freely as well as thermal conductivity of each cell. Then, the only problem is to obtain the weights.

$$
\mathrm{T}(\mathrm{i}, \mathrm{j})=\sum_{\mathrm{k}=1}^{4} \mathrm{p}_{\mathrm{k}} \mathrm{T}_{\mathrm{k}}(\mathrm{i}, \mathrm{j})
$$

where $T(i, j)$ is the temperature at $(i, j)$ node and $T_{k}(i, j)$ denotes $k$-th neighbour of $(i, j)$ node, $p_{k}$ is a proper weight. A sum of all $p_{k}$ 's is always one.

The weights can be obtained using finite difference representation of the differential equation. The final result is (2) [11].:

$$
\begin{aligned}
& \mathrm{T}_{\mathrm{i}, \mathrm{j}}=\left[\mathrm{p}_{\mathrm{e}} \mathrm{T}_{\mathrm{i}+1, \mathrm{j}}+\mathrm{p}_{\mathrm{w}} \mathrm{T}_{\mathrm{i}-1, \mathrm{j}}+\mathrm{p}_{\mathrm{n}} \mathrm{T}_{\mathrm{i}, \mathrm{j}+1}+\mathrm{p}_{\mathrm{s}} \mathrm{T}_{\mathrm{i}-1, \mathrm{j}+1}+\mathrm{S}_{\mathrm{i}, \mathrm{j}} \text { HGEN }\right]_{\frac{1}{\mathrm{t}}}^{1} \\
& \mathrm{p}_{\mathrm{e}}=\frac{\Delta \mathrm{y}}{2 \Delta \mathrm{x}}\left(\mathrm{k}_{\mathrm{i}, \mathrm{j}}+\mathrm{k}_{\mathrm{i}, \mathrm{j}-1}\right) ; \mathrm{p}_{\mathrm{w}}=\frac{\Delta \mathrm{y}}{2 \Delta \mathrm{x}}\left(\mathrm{k}_{\mathrm{i}-1, \mathrm{j}}+\mathrm{k}_{\mathrm{i}-1, \mathrm{j}-1}\right) ; \mathrm{p}_{\mathrm{n}}=\frac{\Delta \mathrm{x}}{2 \Delta \mathrm{y}}\left(\mathrm{k}_{\mathrm{i}-1, \mathrm{j}}+\mathrm{k}_{\mathrm{i}, \mathrm{j}}\right) ; . \\
& \mathrm{p}_{\mathrm{s}}=\frac{\Delta \mathrm{x}}{2 \Delta \mathrm{y}}\left(\mathrm{k}_{\mathrm{i}-1, \mathrm{j}-1}+\mathrm{k}_{\mathrm{i}, \mathrm{j}-1}\right) ; \mathrm{t}=\mathrm{p}_{\mathrm{e}}+\mathrm{p}_{\mathrm{w}}+\mathrm{p}_{\mathrm{n}}+\mathrm{p}_{\mathrm{s}}
\end{aligned}
$$

where HGEN is a heat generation rate, and $S_{i, j}$ is the heat generation area.

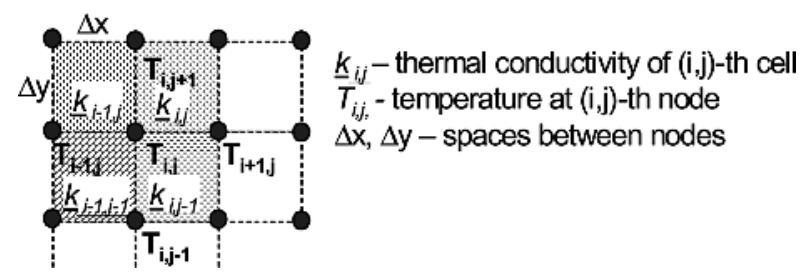

Fig. 5. Every node has its own temperature which depends on temperatures of adjacent cells. Such local relation is typical for cellular automata.

For the isothermal boundary condition (b.c.) we fix the temperature at the boundary node. If the edge is adiabatic, an image of the node which is nearest to the edge is placed, the other side of boundary is to obtain a reflective property of adiabatic b.c.

The structure and the temperature distributions are shown in Fig. 6. The structure resolution is 100x100 pixels, and each pixel represents one cell. The shade indicates material.

To calculate the steady-state distribution we needed ca. 10 minutes (Athlon XP, $2800+$ ). The effective thermal conductivity was found about $90 \mathrm{Wm}^{-1} \mathrm{~K}^{-1}$.

The volume ratios were respectively: $\mathrm{cBN}-83 \%$, glass $-7 \%$, air $-10 \%$. This is much closer to the values placed in Table 1, but also close to the measurements. However the lower bound of glass phase is still limited by the size of one pixel. For 100 pixels we cannot decrease it below $1 \%$ if any. And each grain must be bounded by the glass. To decrease the volume fraction of the glass one should have to increase the resolution which would increase time of calculation. For 200x200 pixels 2 hours (Athlon XP, 2800+) were necessary to find the final solution. 

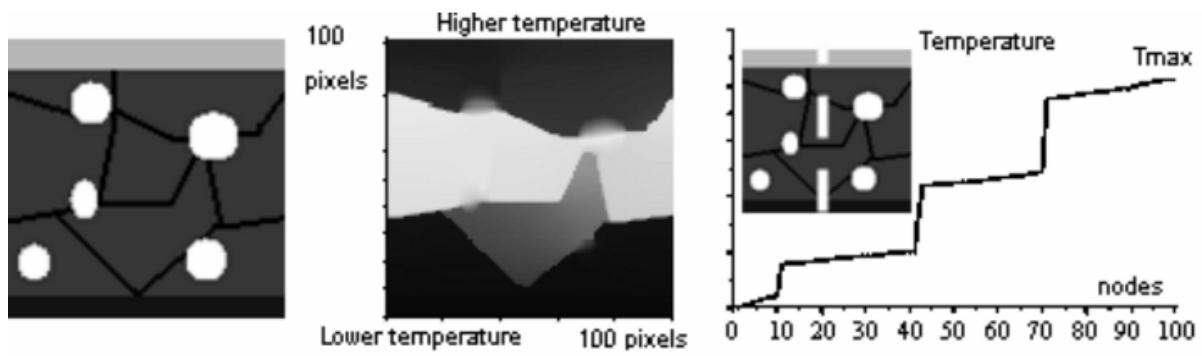

Fig. 6. The 100x100 pixels structure, the temperature distribution and the temperature profile along dashed line. The size of each pixel was assumed $1 \mu \mathrm{m} \times 1 \mu \mathrm{m}$.

So we have to deal with two problems: we have to (i) decrease time of computation by lowering the resolution (keeping in mind the accuracy), (ii) decrease the lower limit of the glass volume. This can be done by a slight modification of the CA

\section{Modified Cellular Automata Approach}

Having in mind the temperature distribution (Fig. 6), and the temperature profile, one can see that the temperature steps correspond to the glass regions and the profile reminds a profile with contact thermal resistances. Thus one can eliminate pixels which surround each grain by introducing contact resistances.

The formulas (2) were derived from the finite difference, but they can also be obtained by connecting adjacent nodes by thermal resistors. Introducing thermal contact resistances the resistances can be calculated from the conductivity and dimensions of a cell Fig. 7 .

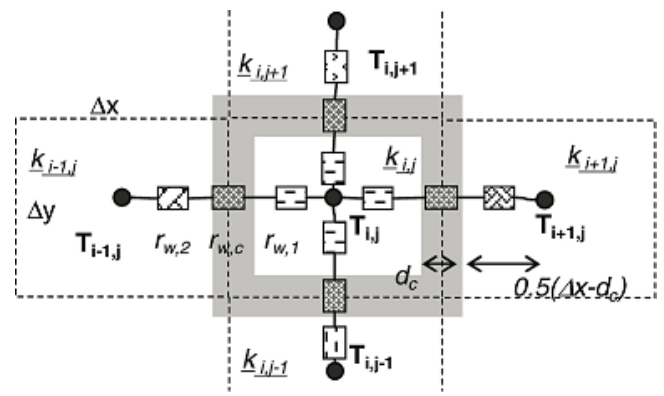

Fig. 7. The way of introducing contact thermal resistance. $d_{c}-$ thickness of the contact. Please note that here the node lies at the center of the cell.

The resistance of the contact is calculated as the resistance of a layer with the thickness $d_{c}$.

$$
\mathrm{r}_{\mathrm{w}, \mathrm{c}}=\frac{1}{\mathrm{k}_{\mathrm{c}}} \frac{\mathrm{d}_{\mathrm{c}}}{\Delta \mathrm{y} \cdot[1 \mathrm{~m}]}
$$


Assuming that the thickness of a glass layer is small and that its thermal conductivity is much lower than the conductivity of c-BN one can assume that there is no heat conduction along the joint layer and the resistance $r_{w, 1}$ is:

$$
\mathrm{r}_{\mathrm{w}, 1}=\frac{1}{\mathrm{k}_{\mathrm{i}, \mathrm{j}}} \frac{\Delta \mathrm{x}}{2\left(\Delta \mathrm{y}-\mathrm{d}_{\mathrm{c}}\right) \cdot[1 \mathrm{~m}]} .
$$

Similarly we obtain other resistances and then proper weights. So the final recipe for the diffusive CA has not changed, but the value of weights was modified.

Two temperature profiles in two composite films are presented in Fig 8 . The composition of the films a) and b) is: c-BN $-91 \%$ and $88 \%$, glass $-1 \%$ and $3 \%$, air $8 \%$ and $8 \%$ of volume ratio. The effective thermal impedance was about $773 \mathrm{Wm}^{-1} \mathrm{~K}^{-1}$ and $515 \mathrm{Wm}^{-1} \mathrm{~K}^{-1}$, respectively. We can see that for the b) structure the steps are much higher since the thickness of the glass film is higher. Even a small change of glass content may change the effective conductivity significantly. For this method of modeling there is no bottom limit for glass fraction.
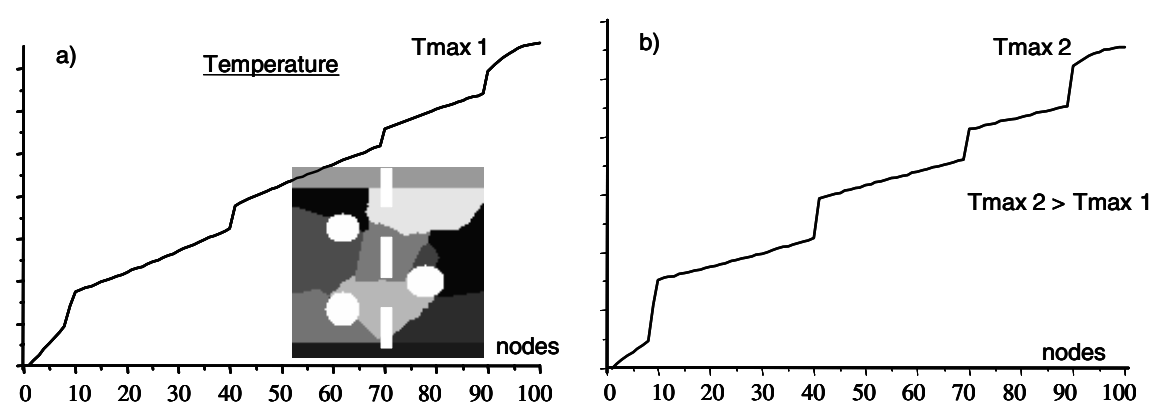

Fig. 8. Temperature profile along dashed line. The domain consists of 100x100 pixels of a size $1 \mu \mathrm{m} \times 1 \mu \mathrm{m}$ each. The thickness of contact layer was a) 0.1 and b) 0.3 of a cell size.

\section{Discussion on Efficiency of Cellular Automata}

The system which has been analyzed by means of CA can be also solved by forming a system of linear equations:

$$
\mathrm{A} \bullet \mathrm{X}=\mathrm{B} \text {. }
$$

For the domain of $100 \times 100$ pixels $\mathrm{X}$ - is a vector of $10^{4}$ unknown temperatures. A is a $10^{4} \times 10^{4}$ sparse matrix. $\mathrm{B}$ is a $10^{4}$ elements vector. When using CA we need at least one 100x100 matrix where we store temperatures and four 100x100 matrices for weights. So we do not need a $10^{4} \times 10^{4}$ matrix. Even if it's a sparse matrix we have to remember indeces of non zero elements. The simplest iterative methods of solving (5) are the Jacobi and Gauss-Seidel algorithms [12]. In both cases we have not obtained shorter time of computation in comparison to CA algorithm. It doesn't mean that CA methods are fast enough. The speed is a problem, but a problem which can be solved. First we can use a non-uniform grid: finer near grain boundaries and courser inside a 
grain. This will affect only weights. Moreover, different levels of mesh could be introduced so an approximated solution could be found first, and next, using more accurate mesh a detail solution would be obtained.

\section{Conclusions}

The purpose of this study was to investigate an efficient method of accurate modeling of composites' thermal properties. We have considered: (i) an approach which utilizes ANSYS software, (ii) a cellular automata method.

Using the first approach, the result comes very fast but if we have the geometry already prepared. And the basic problem is to generate the structure. We should consider not only the position of each grain but also a possible sticking one grain to another if we need to lower the glass fraction. So should be ready to face up a problem of modeling fabrication process.

Of course this can be the case for CA approach. However we can use a scanned and reviewed image of the composite layer as input data. Since a CA can easily deal with complex boundaries we are not restricted to simple shapes which are necessary for ANSYS. The CA is an easy approach and very intuitive.

\section{References}

1. J.L., German R.M., Hems K.F., Guiton Th.A., Injection Molding AlN for Thermal Management Applications, Bull. Am. Ceram. Soc. 75[8], 1996, pp. 61-65.

2. Mirkarimi P.B., McCarty K.F., Medlin D.L., Review of Advances In Cubic Boron Nitride Symthesis, Materials Science and Engineering, R21 (1997), pp. 47-100

3. Giellissee P. Niculescu H., Temblay J., Achmatowicz S., Jakubowska M., \& others, High Thermal Conductivity Cubic Boron Nitride Thick Films, Proc. of 2001 Int. Symp. on Microelectronics, Oct. 9-11, 2001, Baltimore, pp.379-383.

4. Achmatowicz S., Zwierkowska E., Wyżkiewicz I., Łobodziński W., New Approach to Thermal Conductivity of Thin Films Measurements by Means of Comparative Method, Proc. of $35^{\text {th }}$ Int. Symp. On Microel., 2003, pp. 655-660

5. Gerenrot D., Berlyand L., Philips J.: Random Network Model for Heat Transfer in High Contrast Composite Materials, IEEE Trans. on Advanced Packaging, vol. 26, No. 4, Nov. 2003, 410416

6. Devpura A., Prasher R. S.: Percolation Theory Applied to the Analysis of Thermal Interface Materials in Flip-Chip Technology, Itherm 2000, May 24-26, 2000, Las Vegas, $\mathrm{NV}, 21-28$

7. Staggs J.E.J.: "Estimating the thermal conductivity of chars and porous residues using thermal resistor networks" Fire Safety Journal 37 (2002) 107-119

8. http://scilabsoft.inria.fr/

9. Wolf-Gladrow D.: Lattice Gas Cellular Automata and Lattice Boltzmann Models: An Introduction, Lecture notes in mathematics 1795, Springer-Verlag 2000

10. Wolfram S.: A new kind of science, Wolfram Media (May, 2002)

11. Haji-Sheikh A. Monte Carlo Methods in In Handbook of Numerical Heat Transfer, John Wiley \& Sons, New York, 1988, pp. 673-722

12. Jon H. Mathews, Kurtis D. Fink Numerical Methods Using Matlab $3^{\text {rd }}$ ed., Prentice Hall '99 